\title{
Research on the Fluid-solid Coupling of Wind Turbine
}

\author{
Qiuyun $\mathrm{Mo}^{1,2}$, Shunkang Yan², Junjian Shi ${ }^{2}$, Fei Deng ${ }^{2}$, \\ XiChang Liu $^{2}$ \& Yongqing Yin ${ }^{2}$ \\ ${ }^{1}$ Guangxi Experiment Center of Information Science, Guilin University of Electronic Technology, \\ Guilin, 541004, China \\ ${ }^{2}$ Department of Electrical and Mechanical Engineering, Guilin University of Electronic Technology, \\ Guilin, 541004, China
}

Keywords: wind turbine; blade structure; fluid-solid coupling; finite element.

\begin{abstract}
Researching on fluid-solid coupling problem of wind turbine can help improve the optimization of global structure of wind turbine and improve the level of the blades design, which has been one of the current research hotspots and difficulties in the field of wind turbine design. This paper describes the research status of fluid-solid coupling method, analysis and modelling technology, software applications and other related technical issues in wind power system, expounds in detailed that the key technical troubles and cautions in solving process of wind turbine fluid-solid coupling, discusses the necessity of raising the theoretical level of wind turbine fluid-solid coupling in China.
\end{abstract}

\section{Introduction}

Coupling analysis is interaction analysis between two or more physical fields, nowadays, coupling problem need to be considered with more and more practical issues in the engineering field, and especially in the field of fluid dynamics. Wind turbines run the random flow field, and the load acting on the wind turbine changes by wind shear, yaw, random gust, start and stop of wind turbine and so on, the coupling effects from change of wind load and flexible blade structure will intensify deformation and structural vibration of rotor blade, that influence efficiency and the safe operation of the wind turbine. Under the influence of bad weather, Wind turbine blade profile changes because of grease, dust, snow and other debris on the blade surface, thus the aerodynamic performance of the blade is affected, and the wind turbine power generation efficiency is reduced. Ignoring the coupling won't make blade deviate from the original design.

This paper summarizes the research status of fluid-solid coupling method, analysis and modelling technology, software applications and other related technical issues, and describes the key technical problems and cautions in solving process of wind turbine fluid-solid coupling.

\section{Developing history of fluid-solid coupling}

Fluid-solid coupling problems involve too broad to determine appropriate classification, here takes Applied Mechanics Reviews published by ADME to illustrate the development history of fluid-solid coupling.

From 1970 to 1980, the entry of fluid-structure coupling doesn't appear in AMR classification. From 1981-1983, the fluid-structure interaction appears in AMR classification, but doesn't have detailed subdirectory. Since 1984, AMR classification lists the subdirectory of fluid-structure interaction [1]. Figure 1 shows the statistical curve of the number of abstracts about fluid-solid interaction in AMR every year between 81-95 year, which points out that there is a big jump rise in 84 year, and it still has been an increasing trends after 84 year. The study of fluid-structure coupling dynamics attracts and more attention over time. 


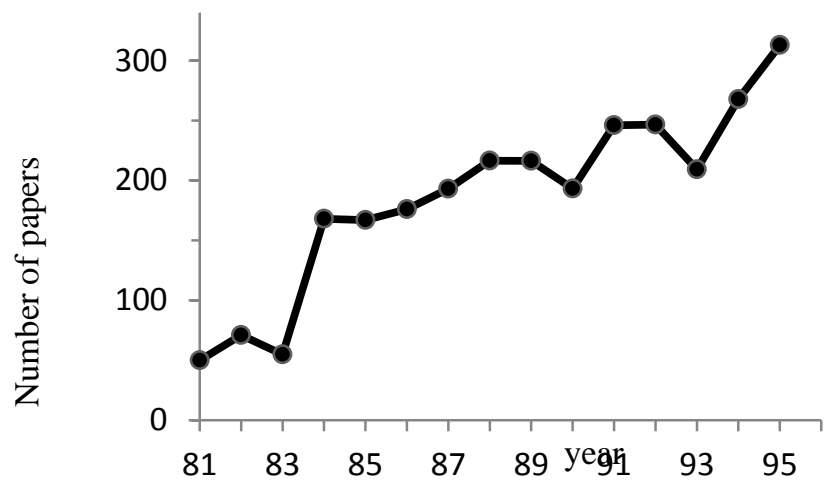

Fig. 1: Statistical numbers curves for fluid-solid coupling papers in AMR of ASME (USA)

The fluid-solid coupling problem of wind turbine blades is the aeroelasticity problem of turbine, the aeroelasticity problem is the changes of displacement, velocity and acceleration of the blades while effected by aerodynamic, inertial force and gravity. Blades produce vibrations that are formed by mutual coupling among waving, Shimmy and torsional vibrations of blades under the effects of forces. Except the vibration coupling, it's necessary to consider the coupling effects between the solid blades and air.

Aeroelastic problem first appears in aeronautical engineering, early in 1913, Brewer [2] analyzes that the wing fracture accident of Langley's first monoplane dynamic test is caused by divergence of aeronautical static torsion. Theordosen [3] establishes non-steady aerodynamic theory on the basis of solving the non-steady aerodynamic force of the control surface airfoil, which make the foundation for aeroelastic instability research. After World War II, Bisplinghoff [4-5], Templeton [6] writes the influential monograph associated with aeroelastic,that shows aeroelastic problem has gradually become an independent discipline. However turbomachinery usually works under the condition of high temperature, high pressure and high speed, so that the cost of test is very high, and some other issues such as stalling flutter is difficult experimented due to safety, above all, we must do research into the theory of fluid-solid coupling problem to guide the design.The lower solution based on interpolation.

\section{Analysis and modelling technology of fluid-solid coupling}

Fluid-solid coupling solution. The general solution is to put the solid as main vibration object, and take the effect of the fluid as a damping (additional resistance), then solve the problem of fluid flow based on solid vibration conditions. Another method is the use of substructure (or modal synthesis) method, the solid and fluid are treated as two substructures in a system, establishing their models, taking the interaction force as the generalized coordinates in the fluid-solid phase boundary, and reducing coordinates necessarily to get easy vibration problem-solving model of fluid-solid coupling system. In general, these methods are using approximation theory and statistical method by reason of computer restriction.

With the rapid development of computer and nonlinear dynamic disciplines, a powerful tool is used for analysis of fluid-solid coupling. Fluid-solid coupling is a typical nonlinear dynamical system, which usually can use one or a set of nonlinear differential equations to describe and solve these equations with qualitative methods: phase-plane method and quantitative approach. Quantitative approach includes analytical method and numerical method. Analytical method is only used in exceptional circumstances and numerical method can be adopted more often than not. Common numerical method has FEM method and BEM method. FEM method is suitable for solving statics and dynamics problems of large and complex structure, BEM method is a new distinctive numerical method after FEM method, which puts the differential equation boundary 
value problems of elastic into boundary integral equation, and develops with discrete numerical technology of FEM method.

In the study of coupling problem, the biggest difficulty is the use of a unified coordinate and the coordination of the two-phase interface. Because Lagrange coordinate system focuses on a particle in solid mechanics, but Euler coordinate system focuses on spatial point in fluid mechanics. For small campaign issue this difference can be ignored, but for big ones must be distinguished, compared with single solid or fluid nonlinear problems, how to build the interface coordinate is the peculiar difficulty for nonlinear coupling problem. Hirt [7] proposes ALE method used in fluid flow computation to calculate for Fluid-solid coupling analysis [8-10], and solves problems between coordination and free surface interface, some authors have used FEM method and BEM method. ALE coordinate system can be arbitrary velocity in space motion, when the velocity is zero it is Euler coordinate system; when the speed is equal to the particle velocity it will be the Laplace coordinate system. So ALE system provides a unified description of the two coordinate systems, but it is still very difficult to take a combination of both the effective way for solving nonlinear coupling problem.

Modelling technology.FEM method that solving complex problems such as fluid dynamics is an effective method of numerical analysis, currently large analysis software commonly used has ANSYS, Comsol Multiphysics, PATRAN, ABAQUS and so on. Figure 2 shows the general steps for wind turbine blade structure finite element analysis.

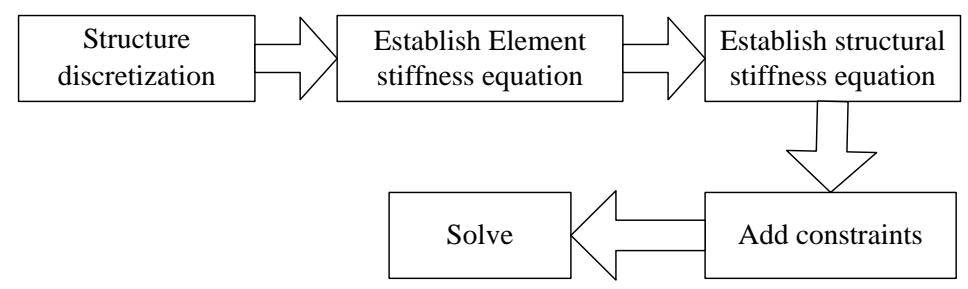

Fig. 2 General basic flow of blade structure finite element

Fluid-solid coupling simulation needs governing equations of fluid. Turbulent flow is a common flow phenomenon in nature, turbulence model frequently used can be classified according to the number of differential equations: zero equation model, one-equation model, two-equation model, four-equation model, seven-equation model and so on. For simple fluid motion, the accuracy generally gets to be higher with the number of increasing equations except complex turbulent motion. Two equations model is most widely used in engineering, the basic two-equation model is the standard k- $\varepsilon$ model, which is introduced on the equation of turbulent kinetic energy $\mathrm{k}$ and dissipation rate $\varepsilon$, Haiping Chen [11] calculates the strain distribution of the fluid-solid coupling on the blades according to the k- $\varepsilon$ turbulence model, draws the law and force distribution pattern of wind turbine blades, and the result is consistent with the actual situation. N-S equations and k- $\omega$ turbulence equations don't be used because it is most convenient for using $k-\varepsilon$ equation to describe turbulence near the wall region. Standard k- $\varepsilon$ turbulence model:

$$
\begin{gathered}
\frac{\partial(\rho \kappa)}{\partial \mathrm{t}}+\frac{\partial\left(\rho \kappa u_{i}\right)}{\partial x_{i}}=\frac{\partial}{\partial \mathrm{x}_{i}}\left[\left(\mu+\frac{\mu_{t}}{\sigma_{k}}\right) \frac{\partial k}{\partial x_{j}}\right]+\mathrm{G}_{k}-\rho \varepsilon \\
\frac{\partial(\rho \kappa)}{\partial \mathrm{t}}+\frac{\partial\left(\rho \kappa u_{i}\right)}{\partial \mathrm{x}_{i}}=\frac{\partial}{\partial \mathrm{x}}\left[\left(\mu+\frac{\mu_{t}}{\sigma_{\varepsilon}}\right) \frac{\partial \varepsilon}{\partial x_{j}}\right]+\frac{C_{1 \varepsilon} \varepsilon}{k} G_{k}-C_{2 \varepsilon} \rho \frac{\varepsilon^{2}}{k}
\end{gathered}
$$

Many literatures detail the modelling method of fluid-structure coupling. Zhang Zhang [12] describes the theory and method of fluid-solid coupling calculation, introduces the method of using ANSYS/CFX to simulate multi-step coupling and does the simulation analysis. Xiaowei Zhang [13] in BUAA uses ANSYS to calculate NASA67 bending vibration modal, then calculates blade aerodynamic forces and damping with amplitude given in the flow field. But there has been a general lack of researching on the factors of tip clearance and blade angle of attack affecting the stability. Danmei Hu [14] uses ANSYS workbench to obtain that angle of attack and torque are increased by fluid-solid coupling, the increasing angle of attack is the main cause of increasing torque. Yuan Li [15] does the fluid-solid coupling calculation of 2.5MW wind turbine, shows that 
the angle of attack increases at the same time when the blades have a greater wielding, the axial thrust and torque also increase slightly. Considering that increasing blade pneumatic angle of attack caused by blade twisting will make the calculation of the torque value be more closed to the design value. It is observed that the effective fluid - solid coupling modelling needs to consider the chance of aerodynamic performance parameters of wind turbine.

\section{Software applications}

Many experts research on the finite-element analysis of wind turbine fluid-solid coupling at home and abroad, but most are based on ANSYS software. COMSOL Multiphysics has efficient computational performance and unique multi-field coupling analysis capability, which ensures highly accurate numerical analysis [16]. It provides a friendly graphical user interface and convenient modelling tool that can be directly used in COMSOL fluid-structure coupling module, then users can set static or transient calculation needed for multi-physics modelling and simulate. Qian Sun [17] uses COMSOL to do the modal analysis of wind turbine tower under loads of wind and wave, and determines that the cause of the tower resonance depends on the low-order frequencies of the tower under the external excitation frequency. ANSYS is widely used by accumulating a lot of models and experiences, and COMSOL also develops rapidly due to the powerful capability of solving multi-field coupling problem.

\section{Conclusions}

The most basic way to describe fluid-solid coupling is mathematical method. To research on wind turbine fluid-solid coupling deeply, researchers must study and learn to master every math method of modern classical dynamics, and the latest research results in mathematics can be applied in the study of fluid-solid coupling system, meanwhile learn from the computer processing technology. Discussing the wind turbine fluid-solid coupling from the mechanism and essence can greatly shorten the design cycle of blade design and also can improve the accuracy. At present domestic research of wind turbine fluid-solid coupling focus on the 2D model, which still has much room for development, researchers need to further improve and build new mathematical model in the engineering application of wind turbine, improve fluid-solid coupling theory to a higher level.

\section{Acknowledgements}

The research work was supported by Natural Science Foundation of China under Grant No. 51465010 and GuangXi Key Laboratory of Manufacturing System \& Advanced Manufacturing Technology under Grant No. 14-045-15-010Z.

\section{References}

[1] Z. Wang, H. Wu \& H.J. Jia, The Development of Numerical Approach Method of Fluid-structure Interaction Mechanics and the Application of Commercial Softwares. Machine Tool \& Hydraulics, 36(4),pp.192-195,2008.

[2] J.T Xing, S. Zhou \& R.J Cui, A SURVEY ON THE FLUID-SOLID INTERACTION MECHANICS. Advances in Mechanics,27,pp.19-38,1997.

[3] T. Theodore, General theory of aerodynamic instability and the mechanism of flutter. NASA Technical Reports Server (NTRS),20,pp.413-433,1935

[4] R.L. Bisplinghoff \& H. Ashley, Principles of aeroelasticity. Phoenix, U.S.A Dover Pubns,2002.

[5] R.L. Bisplinghoff, H. Ashley \& R.L. Halfman, Aeroelasticity. Phoenix, U.S.A Dover Pubns,1996. 
[6] H. Templeton, A review of the present position on flutter. Washington: Third Meeting of the Structures and Materials Panel,1956.

[7] C.W. Hirt, A.A. Amsden \& J.L. Cook. An arbitrary Lagrangian-Eulerian computing method for all flow speeds. J. of Comp. Phys.,14,pp.227-253,1974.

[8] D. J. Arbitrary, Lagrangian-Eulerian finite element method. in: Belytschka T, Hughes J R eds. Computational Methods for Transient Analysis. Elsevier Science Publishers, Netherlands, pp.473-516,1983.

[9] Z.H. Liu \& Y.Y Huang. An arbitrary Lagrangian-Eulerian boundary element method for large-amplitude sloshing problem. In: Du Q H ed. Theory and Applications of Boundary Element Methods. International Academic publishers, Beijing,pp.153-160,1991.

[10] Y.Y. Huang, etal, Fluid-structure impact analysis with a mixed method of arbitrary Lagrangian-Eulerian BE and FE. Acta Mechanica Solida Sinica,6(4),pp.365-376,1993.

[11] H.P. Chen, W.L. Sun \& J. Guo, Fluid-solid Coupling Analysis on Wind Generators Blade. Machine Tool \& Hydraulics,19,pp.19-82,2010.

[12] Z. Zhang, Research on Dynamic Stability of Blade Structure Based on Fluid-Structure Interaction. Nanjing University of Aeronautics and Astronautics,2012.

[13] X.W. Zhang, Y.R. Wang \& X. Zhang, Numerical method for fluid-structure interaction in turbomachinery bladings. Journal of Aerospace Power,24(7),pp.1622-1626,2009.

[14] D.M. Hu, Z.C. Zhang, K. Sun \& J.P. Zhang, Computational Analysis of Wind Turbine Blades Based on Fluid-structure Interaction. Proceedings of the CSEE,17,pp.98-104+18,2013.

[15] Y. Li, Numerical Simulation of Fluid-Structure Coupling of 2.5MW Wind Turbine Blades. JOURNAL OF ENGINEERING THERMOPHYSICS,34(1),pp.71-74,2013.

[16] Y. Wang, Research on the test and theory of strength between film bearing babbitt and steel. Taiyuan University of science \& Technology,2014.

[17] Q. Sun, L.B. Zhu, Z.S. Zhu \& C.Y. Ji, Modal analysis of offshore wind turbine tower based on Comsol Multiphysics. Machinery Design \& Manufacture,8,pp.204-206,2011. 